\title{
Self-organized Evacuation Based on LifeBelt
}

\author{
Kashif Zia and Alois Ferscha \\ Institute of Pervasive Computing, \\ Johannes Kepler University Linz, \\ Altenberger Strasse 69, A-4040 Linz, Austria \\ \{kashif, ferscha\}@pervasive.jku.at \\ http://www.pervasive.jku.at
}

\begin{abstract}
In this paper, we have investigated the feasibility of a selforganized evacuation process when compared with a centralized control. The evacuation strategy is based on 'predicted exit time' (a relation of 'estimated time to reach to an exit', 'exit capacity' and 'exit population') for each of the exit in a multi-exit environment, selecting the minimum value exit. The self-organized strategy is based on information propagation in a peer-to-peer fashion, initiated by a special agent in each of the exit area. The propagation range ('zone of influence') is dependent on intensity and direction of peers interaction. Based on the propagated dataset, each agent can make an autonomous decision, conceptually a converse of centralized strategy where each agent is directed by a server. The evacuation process in supported by a wearable device, i.e. LifeBelt. Through large scale simulations using cellular automata technique and a challenging airport terminal model, we have proved that an efficient evacuation based on principles of self-organization is a real possibility, even in an infrastructureless environment.
\end{abstract}

Keywords: Self-organized evacuation simulation.

\section{Introduction}

Several modeling approaches are applied to study crowd dynamics separately and in combination [1. However, the most important approaches are: Cellular Automata (CA), Lattice Gas (LG), Social Force (SF), and Fluid Dynamics (FD) models. For the sake of this paper, the reason for choosing $\mathrm{CA}$ as a modleing choice its intrinsic capability to model basic structures and hence the global space, in conjunction with behavioral rules, along with its simplicity. A CA model is based on a regular grid of the cells in which each cell is occupied by a single individual. The space (grid occupancy) and local (neigborhood state) rules in combination, describe the next cells an agent needs to occupy at each time stamp. Considering that an individual knows his direction of motion (towards an exit), the strategy of choosing the next cell to move to depends on the intensity and scope of his perception in the direction of the exit. In more technical terms, intensity is defined as individual's ability to interact with other individuals in all directions, whereas scope is defined as individual's ability to interact with a

T. Spyropoulos and K.A. Hummel (Eds.): IWSOS 2009, LNCS 5918, pp. 250-255, 2009.

(C) IFIP International Federation for Information Processing 2009 
only a subset of it. For example, it may be assumed that a normal vision allows an individual to see the state of the cells within an intensity equal to three cells (all cells within three cells radius). Moreover it can be assumed that, due to limitations incurred by human vision, an individual can only see what lies reasonably ahead (in the line of sight), hence limiting the scope of cells he can view. Combining intensity and scope, we have termed the interaction range of an individual as his Zone of Influence $(\mathrm{ZoI})$.

In contrast with most of the CA mechanism, we focus on three aspects of an evacuation situation, overlooked by most of the research community. The first difference lies in not considering an evacuation environment as a normal structure with no breakdowns. For example, in an emergency situation it is hard to assume that there would be no power failure due to structural collapse. If that is the case in reality, there would be no vision (particularly at night) to view any thing within ZoI. Secondly there is little effort in designing a strategy for optimal exit selection (in a multi-exit environment) based on factors other than distances to the exits. For example, authors in [2] have presented a strategy of selecting an exit based on occupants' density (Exit Population (EP)) in an area of interest (Exit Area (EA)) around each of the exit, in conjunction the with distance. There are two issues in this model. (i) The model is based on human perceptions and does not account for limited or no visibility. It is 'assumed' that individuals are aware of the exit area activities, irrespective of the exit distance. (ii) For optimal exit selection, the model does not consider the Exit Capacity (EC) the width or capacity an exit has. We already designed a more efficient strategy [3] and used it in this paper (an implementation in a more complex environment). Our model is based on EP, EC and Time to reach to an Exit Area (TEA). It is designed for environments with no visibility and hence rely on two-way communication mechanism between server and individuals. Still it can be useful in unlimited special cases in an environment where normal human perceptions are active, for example, assisting handicapped people in evacuation, or assisting in a very large unknown environment. Lastly, there are self-organized data dissemination strategies 4 but there is no evidence of its usage in crowd evacuation simulations. Through this paper, we have introduced a novelty by extending Ferscha's model [3] for a truly distributed setting with no centralized server.

For all the three principles highlighted in the paragraph above, there is a need to equip each individual with a helping device having following capabilities:

- It should provide the neighborhood sensing to assist individuals with taking microscopic next cell decision in the dark. The aspects of neighborhood sensing absolutely desirable in this context are distance and orientation, so that it can fill in the requirements for intensity and scope respectively.

- It should provide location coordinates to the centralized server to be used in optimal exit selection mechanism.

- It should provide a peer-to-peer communication mechanism to help disseminating the information to be used in a self-organized evacuation strategy. 
We have designed a wearable helping device, LifeBelt [3], fulfilling all these requirements. Using LifeBelt, optimal exit selection can either be applied in a centralized or distributed manner. At local level, a decision to move to the next cell can also be made based on orientation and distance information provided by LifeBelt, keeping the individual aggregatively inline with the directional guidance provided by the server or extracted from the self-organized data dissemination. Additionally, LifeBelt provides vibro tactile stimulus in the hip area thus keeping the evacuee attentive throughout the evacuation process or to guarantee a successful evacuation from areas with no visibility.

\section{Optimal Exit Selection Centralized (OESC) Mechanism}

The optimal exit selection strategy chooses the minimum value of Predicted Exit Time (PET) between the PET values for all the exits. PET is based on three parameters - the Exit Population (EP), the Exit Capacity (EC) and the Time to reach to an Exit Area (TEA) and is calculated as:

$$
P E T_{i}=T E A_{i}+\left(E p_{i} / E C_{i}\right)
$$

where subscript i represents a typical exit. The following pseudo code represents the mechanism of evacuation executed in each time stamp, in a centralized setting.

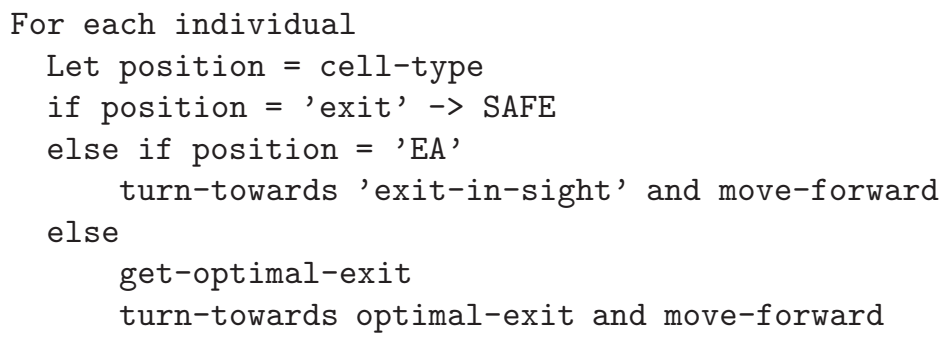

For each of the individual 'position' is the variable which represents the cell type on which an individual is residing. If position is 'exit', it means that the individual has reached to an exit and is safe. If position describes as if individual being in an exit area (EA), the individual can see the 'exit-in-sight' and has to direct towards the corresponding exit by turning towards the exit in sight and moving forward. Finally, if individual is neither at exit or within an EA, it relies on guidance for optimal-exit provided by the central server. After application of eq 1 for each of the exit, the cental server points towards 'optimal-exit' (get-optimal-exit) thus enabling the individual to perform a turn towards corresponding exit and then moving forward. A more detailed discussion of local cell selection mechanism adopted for 'move-forward' is presented in [5] and [3].

\section{Optimal Exit Selection Distributed (OESD) Mechanism}

The mechanism needs a special agent in each EA, which collects the presence of individuals in an exit area. This information is propagated towards the exterior 
individuals in a peer-to-peer fashion. After each round of propagation initiated by the special agents, each individual can perform a single step motion. The following pseudo code represents the mechanism of evacuation executed in each time stamp, in a distributed setting:

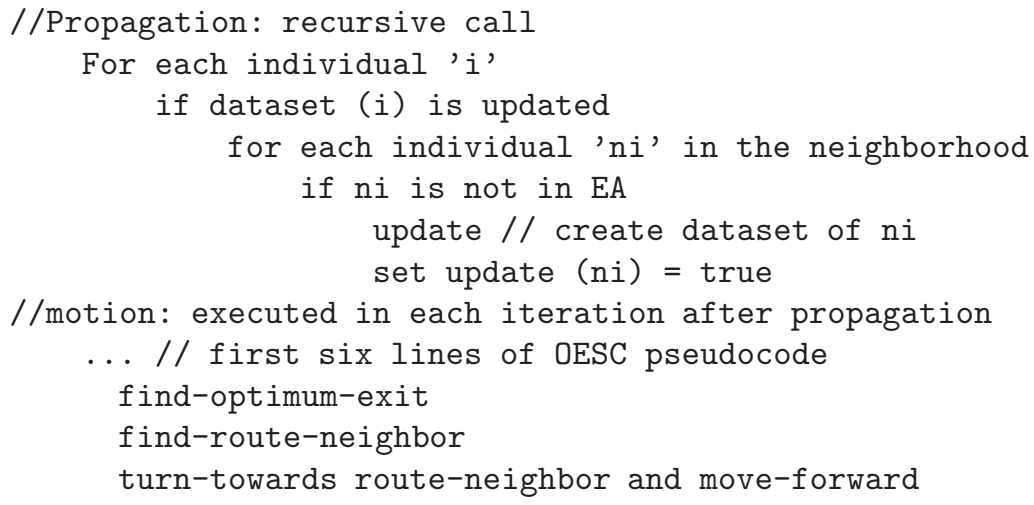

In the propagation phase, each individual recieves a dataset from neighbors. If the dataset recieved is updated, it is propagated to the neighbors within ZoI of the propagating individual if and only if the target neighbror is not in EA. The process terminates when number of individuals updated in a recursive step are zero. The pseudocode for motion is same as that of centrallized case, except that the optimal-exit is calculated by each individual from the information recieved from the neighorhood (find-optimum-exit) and is not directed by central server. After 'finding' the optimal exit, the individual gets the direction of the neighbor which points towards the optimal exit (find-route-neighbor), turns towards corresponding neighbor and then moves forward.

\section{Simulation}

The simulation environment (groud terminal of Ibiza airport, not shown to save the space) has overall cellular dimension of $120 * 40$ including the obstacles (escalators, elevators and counters), exits, baggage recalim areas, check-in area and pathways. There were two purposes of the simulation. The first purpose was to investigate the potential of self-organized evacuation, following an optimal exit selection strategy. Whereas the second purpose was to establish the effect of ZoI on the efficiency of self-organized evacuation. To achieve these goals, we performed a large scale simulation for the two evacuation strategies (OESC and OESD) with the same randomly placed set of 500 individuals. Each strategy was tried with four possible values of ZoI: 2.5, 5.0, 7.5 and 10.0. A comparison of time required to evacuate (all realistic individuals) and number of individuals saved between two strategies is shown in Fig. 1 which helps in drawing useful conclusions. Before jumping into conclusions, there are few terms (phenomenan) which require definitions. (i) Migration: an individual changing its destination from one exit to another. Essentially, this happens due to emergence of new 
Time vs. Safe

Zol based comparison

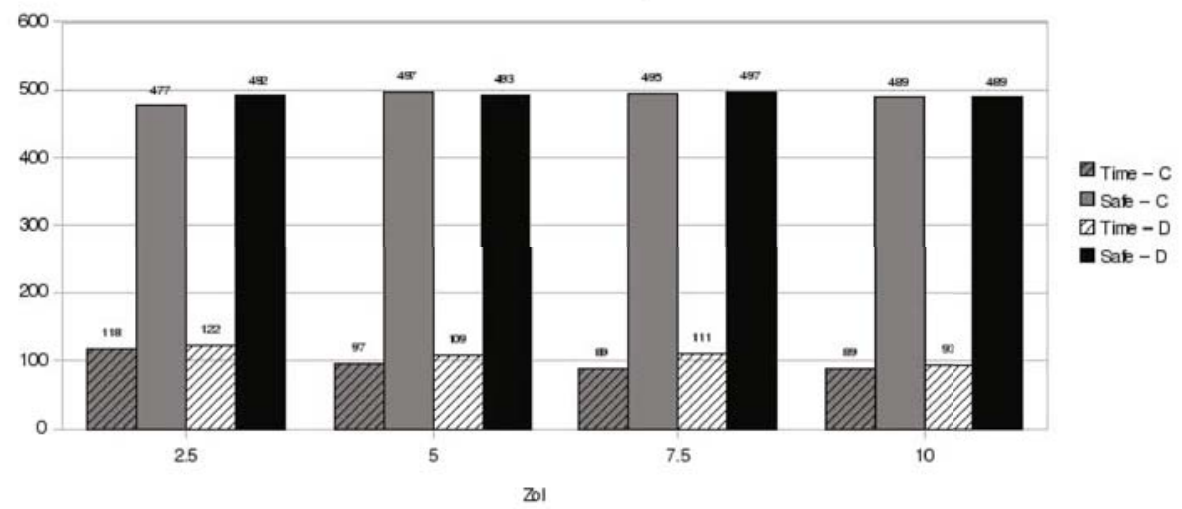

Fig. 1. ZoI based comparison. $\mathrm{C}=$ centralized, $\mathrm{D}=$ distributed.

situations in a surrounding. For example a migration is necessary to balance the load from exits with low EC to exist with higher EC. (ii) Exaustion: a stituation in which there is no more individuals in EA of an exit. Following results are drawn:

1. In case of very small ZoI (2.5) with OESC, the migration starts very late (after 40th iteration), in fact after exaustion of low EC exits. The low EP (due to less $\mathrm{ZoI}$ ) does not allow the factor $\mathrm{Ep}_{i} / \mathrm{EC}_{i}$ to be significant unless the EP becomes zero. That is the reason, with the similar directional guidance and due to limitation in local navigational capabilities, the individuals stuck around obstacles (particularly the ramp beside exit 4). This decreases the number of individuals saved (477). In OESD, the migration phenomena remains the same, but there is an increase in saved quantity (492) due to the reason that the individuals are not provided with absolute direction towards optimal exit. They follow the connected individuals which are connected to the exit by a chain of individuals, hence by passing the obstacles.

2. In case of ZoI equal to 5 with OESC, the migration starts early (around iteration 10). This helps in decreasing the evacuation time and an increase in number of individuals saved. With greater ZoI, it is obvious that there would be late migration as well. In OESD, the pattern is almost the same, except that there is no possibility of late migration due to disconnections. Obviously this decreses the saved quantity from 497 (OESC) to 483 (OESD).

3. In case of ZoI equal to 7.5, both OESC and OESD strategies ensure same number of saved individuals, which is on higher side. Obviously, the migration starts very early and there is alot of fluctuation throughout. In case of OESC, the exaustion is very balanced for all exits. The difference between evacuation time (89 vs. 111) is due to very intense migrations in OESD, which in fact, let the exist with smaller EC finsih first. 
4. A further increase in $\mathrm{ZoI}(\mathrm{ZoI}=10)$ balances the difference in evacuation time (between two strategies), but there is a slight decrement in number of individuals saved (for both strategies). This decrease is environment specific and may not be there in other environments. Typically, a huge ZoI, let the EA of exit 4 extend itself on the other side of the ramp, leaving few individuals there in later stages of simulation.

On top of results presented here (related with effect of ZoI value on the efficiency), it is obvious that self-organized evacuation is not impossible. Although slightly on the lower side, the evacuation efficiency of OESD is certainly comparable with OESC. Additionally, self-organized evacuation based on LifeBelt is the only possible strategy in an infrastructureless environment where human perceptions are not active or overwhelmed.

\section{Conclusion}

In this paper, we have investigated the effects of using LifeBelt on the efficiency of crowd evacuation from a harazdous environment. The factors making an environment hazardous can be a complete blackout, collapse of infrastructure or lack of evacuee attention. In such a situation, a self-organized evacuation strategy remains the only possibility. LifeBelt being an enabling technology provides us with essential features required for performing self-organized evacuation process. Through simulation, We found evacuation efficiency of self-organized strategy comparable with the centralized strategy in a multi-exit environment.

Acknowledgments. This work is supported under the FP7 ICT Future Enabling Technologies pro- gramme of the European Commission under grant agreement No 231288 (SOCIONICAL).

\section{References}

1. Zheng, X., Zhonga, T., Liua, M.: Modeling crowd evacuation of a building based on seven methodological approaches. Building and Environment 44(3), 437-445 (2009)

2. Weifeng, Y., Hai, T.K.: A nove algorithm of simulating multi-velocity evacuation based on cellular automata modeling and tenability conditions. Physica A: Statistical Mechanics and its Applications 379(1), 250-262 (2007)

3. Ferscha, A., Zia, K.: On the Efficiency of LifeBelt based Crowd Evacuation. In: 13th IEEE/ACM International Symposium on Distributed Simulation and Real Time Applications, Singapore, October 25-28, IEEE Computer Society Press, Los Alamitos (2009)

4. Adler, C., Eigner, R., Schroth, C., Strassberger, M.: Context-adaptive Information Dissemination in VANETsMaximizing the Global Benefit. In: Proc. 5th IASTED Int. Conf. on Communication Systems and Networks, p. 712 (2005)

5. Ferscha, A., Zia, K.: LifeBelt: Silent Directional Guidance for Crowd Evacuation. In: Proceedings of the 13th International Symposium on Wearable Computers (ISWC 2009). IEEE Computer Society Press, Los Alamitos (2009) 